ARTICLE

\title{
Chromosome drives via CRISPR-Cas9 in yeast
}

\author{
Hui $\mathrm{Xu}^{1,2}$, Mingzhe Han (1) 1,2, Shiyi Zhou ${ }^{1,2}$, Bing-Zhi Li, ${ }^{1,2}$, Yi Wu (i) ${ }^{1,2 凶}$ \& Ying-Jin Yuan (1) ${ }^{1,2}$
}

Self-propagating drive systems are capable of causing non-Mendelian inheritance. Here, we report a drive system in yeast referred to as a chromosome drive that eliminates the target chromosome via CRISPR-Cas9, enabling the transmission of the desired chromosome. Our results show that the entire Saccharomyces cerevisiae chromosome can be eliminated efficiently through only one double-strand break around the centromere via CRISPR-Cas9. As a proof-of-concept experiment of this CRISPR-Cas9 chromosome drive system, the synthetic yeast chromosome $X$ is completely eliminated, and the counterpart wild-type chromosome $X$ harboring a green fluorescent protein gene or the components of a synthetic violacein pathway are duplicated by sexual reproduction. We also demonstrate the use of chromosome drive to preferentially transmit complex genetic traits in yeast. Chromosome drive enables entire chromosome elimination and biased inheritance on a chromosomal scale, facilitating genomic engineering and chromosome-scale genetic mapping, and extending applications of self-propagating drives.

\footnotetext{
${ }^{1}$ Frontier Science Center for Synthetic Biology and Key Laboratory of Systems Bioengineering (Ministry of Education), School of Chemical Engineering and Technology, Tianjin University, 300072 Tianjin, China. ${ }^{2}$ Collaborative Innovation Center of Chemical Science and Engineering (Tianjin), Tianjin University, 300072 Tianjin, China. ${ }^{凶}$ email: yi.wu@tju.edu.cn
} 
B iased inheritance is a unique phenomenon that allows selfpropagated genetic elements to be transmitted to their progenies with higher frequency than that of normal Mendelian inheritance. Selfish genetic elements such as transposons and homing endonucleases, which have control over their own transmission, can increase their own abundance in species $^{1-7}$. Gene drive is a well-known system utilizing homing endonucleases or CRISPR-Cas9 for generating biased inheritance among population ${ }^{8}$. This system has shown a great capability to transmit desired genes in yeast, insects, and mammals ${ }^{9-16}$. In yeast, a CRISPR-based gene drive was used to verify the concept, efficacy and safeguards of driven genetic alteration by sexual reproduction ${ }^{10,11}$. In addition, by targeting the intron 4 -exon 5 boundary of Anopheles gambiae in a caged experiment, a gene drive achieved $100 \%$ invasion in mosquitoes within 7-11 generations, leading to the sterility of females to prevent the propagation of malaria ${ }^{12}$. Recently, gene drives mediated by CRISPR-Cas9 were also demonstrated in the female germline of mice ${ }^{16}$. In addition, other drive systems such as toxin-antidote CRISPR gene drive system have been designed to transmit target genetic elements by reducing the fitness of progeny without drive elements $^{17-19}$.

Here, we develop a strategy of meiotic drive, called a "chromosome drive", that utilizes chromosomes as drive cassettes to realize biased inheritance of large-scale genetic segments in yeast. We find that specific cleavage via CRISPR-Cas9 near the centromere of a Saccharomyces cerevisiae (S. cerevisiae) chromosome can cause the loss of the entire chromosome and enable chromosome drive. As proof of concept, chromosome X (chrX), which encodes a green fluorescent protein (GFP) or the multigenic violacein pathway, is transmitted among synthetic yeasts via chromosome drive. We also demonstrate the applications of chromosome drives for transmitting specific chromosomal structures and complex genetic traits.

\section{Results}

Chromosome elimination by CRISPR-Cas9 in S. cerevisiae. Centromeres are responsible for chromosome segregation during cell division and are vital for maintaining the stability of chromosomes ${ }^{20-22}$. In $S$. cerevisiae, a point centromere can host an entire chromosome up to the length of several megabase pairs or even an entire genome ${ }^{20,23}$. In this study, we found that an entire yeast chromosome was eliminated by only one double-strand break (DSB) around the centromere via CRISPR-Cas9. In a haploid strain, elimination of any chromosome will make yeast inviable ${ }^{24}$. Thus, all the chromosome elimination experiments in this study were performed on diploid strains. Within synthetic yeast chromosome III (synIII), 186 pairs of dispersed geneassociated PCRTags could be used as a DNA watermarking system to distinguish synthetic yeast chromosomes from wild-type chromosomes (Supplementary Fig. 1) ${ }^{25-27}$. Thus, the PCRTaglabeled synthetic yeast chromosome is an excellent model for characterizing the elimination of the entire chromosome.

For biosafety and efficiency, a molecular containment strategy was applied ${ }^{10}$. We designed a guide RNA (gRNA) targeting a specific sequence (5'-CATTATACGAAGTTATAAGTTGG-3') that is $135 \mathrm{bp}$ from the centromere of synIII, which $\overline{\text { does }}$ not exist in chromosome III (chrIII) of BY4741 or in wild-type yeast. The CRISPR-Cas9-induced DSB was processed by mating haploid strain BY4741 and synIII strain, harboring Cas9 and gRNA plasmids, respectively (Fig. 1a). The results of a PCRTag analysis showed that all 186 synthetic PCRTags of synIII were lost in the tested diploid strain (yHX0295), indicating that the entire synIII was knocked out. All 186 wild-type PCRTags were present in $\mathrm{yHX0295}$, revealing that no homologous recombination (HR) occurred between synIII and chrIII during the chromosome elimination (Fig. 1b; Supplementary Fig. 2). In addition, wholegenome sequencing and Southern blot analysis of haploid control strains and yHX0295 also showed that the entire synIII was eliminated in the CRISPR-containing diploid strains (Fig. 1c and Supplementary Figs. 3 and 4). The mating type of S. cerevisiae was determined by the expression of MATa or MATa at the MAT locus on the sex chromosome synIII. The elimination of synIII in the diploid strain will result in the deletion of MATa at the MAT locus, leading to a mating type switch from Aa to A. To characterize the elimination of synIII further, the mating type of the CRISPR-containing diploid strains was tested by tester A strain and tester a strain. The result showed that $78.78 \%$ of CRISPR-containing diploid strains could mate again with tester a but could not mate with tester A, confirming the elimination of synIII and the capability of a change in mating type in diploid strains by the use of this method (Fig. 1d and Supplementary Table 1).

To assess the relationship between chromosome elimination and the position of DSBs, seven positions on the left arm of synthetic chromosome $\mathrm{X}$ (synX) were chosen to be cut, and elimination of synX was characterized for each position. A synthetic CRISPR-targeted sequence (5'-GTTGCAAATGCTCC GTCGACGGG-3') was inserted 50, 100, 150, $200 \mathrm{bp}, 1,208$, (middle of the left arm of synX) and $415 \mathrm{~kb}$ (end of the left arm of synX) from the centromere of synX. Each modified synX harboring episomal gRNA plasmids was mated with BY4742 harboring episomal Cas9 plasmids. For each position, a total of 360 colonies were analyzed via PCRTagging. The results showed that chromosome-elimination efficiency surpassed $80 \%$ when the specific cleavage occurred within $200 \mathrm{bp}$ from the centromere of synX, while there was no chromosome elimination when the specific cleavage occurred $1 \mathrm{~kb}$ from the centromere of synX (Fig. 1e and Supplementary Table 2). These results indicated that the entire chromosome could be efficiently eliminated through only one DSB near the centromere via CRISPR-Cas9 in $S$. cerevisiae.

Chromosome drives with a synthetic metabolic pathway. Yeast is a model organism whose sexual reproduction can be manually controlled in the laboratory; thus, it is suitable for safeguarding experiments involving self-propagating drive systems ${ }^{10}$. Yeast chromosomes can be individually lost in diploid strains by adding a GAL1 promoter in cis to centromere sequences, and most $S$. cerevisiae $2 n-1$ strains can spontaneously endoreduplicate to $2 n$ strains $^{28}$. Therefore, we designed a proof-of-concept experiment of CRISPR-Cas9 chromosome drive to eliminate synX (harboring a red fluorescent protein (RFP) gene) and duplicate its counterpart wild-type chrX (harboring a GFP gene) via sexual reproduction. Compared with normal inheritance, the chromosome drive system will result in biased inheritance of the desired chromosome and will yield four green spores within one tetrad. In our experiment, the synthetic CRISPR-targeted sequence $\left(5^{\prime}\right.$ GTTGCAAATGCTCCGTCGACGGG-3') was inserted $50 \mathrm{bp}$ from the centromere on the left arm of synX, and the RFP gene was inserted $156 \mathrm{~kb}$ from the centromere on the right arm of synX in strain yHX0299, while the GFP gene was inserted $320 \mathrm{~kb}$ from the centromere on the left arm of chrX in strain yHX0141. The two strains described above were mated to initiate the process of chromosome elimination. After sporulation of the mated diploid strains, the fluorescence of the tetrads was observed via a fluorescence microscope. The results showed that all four spores of the observed tetrads displayed green fluorescence and no red fluorescence, while the control group of undriven tetrads displayed two green spores and two red spores. These results 
a

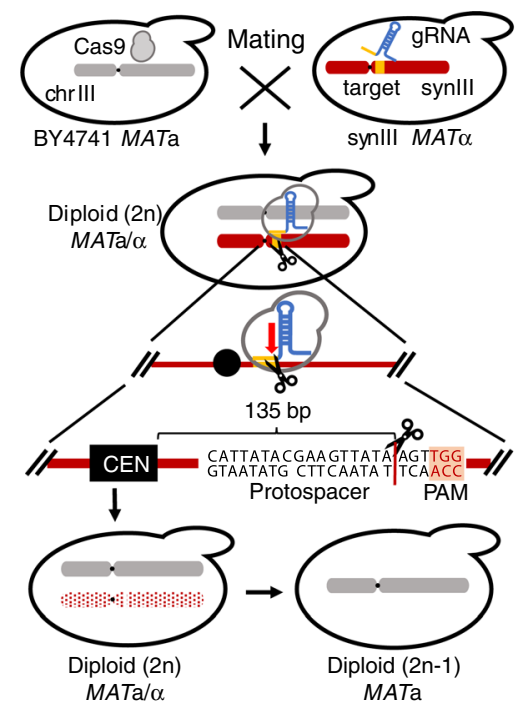

C

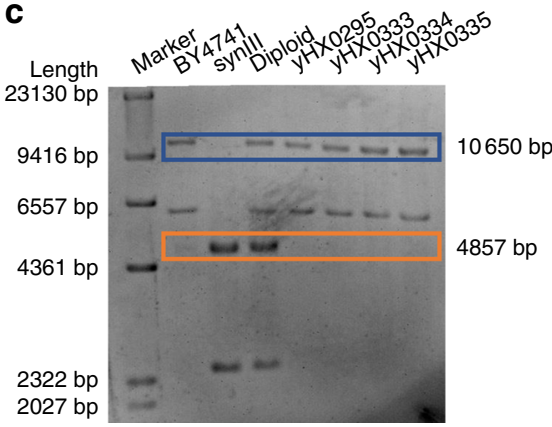

b Chromosomal

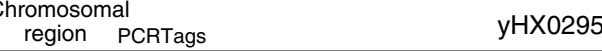

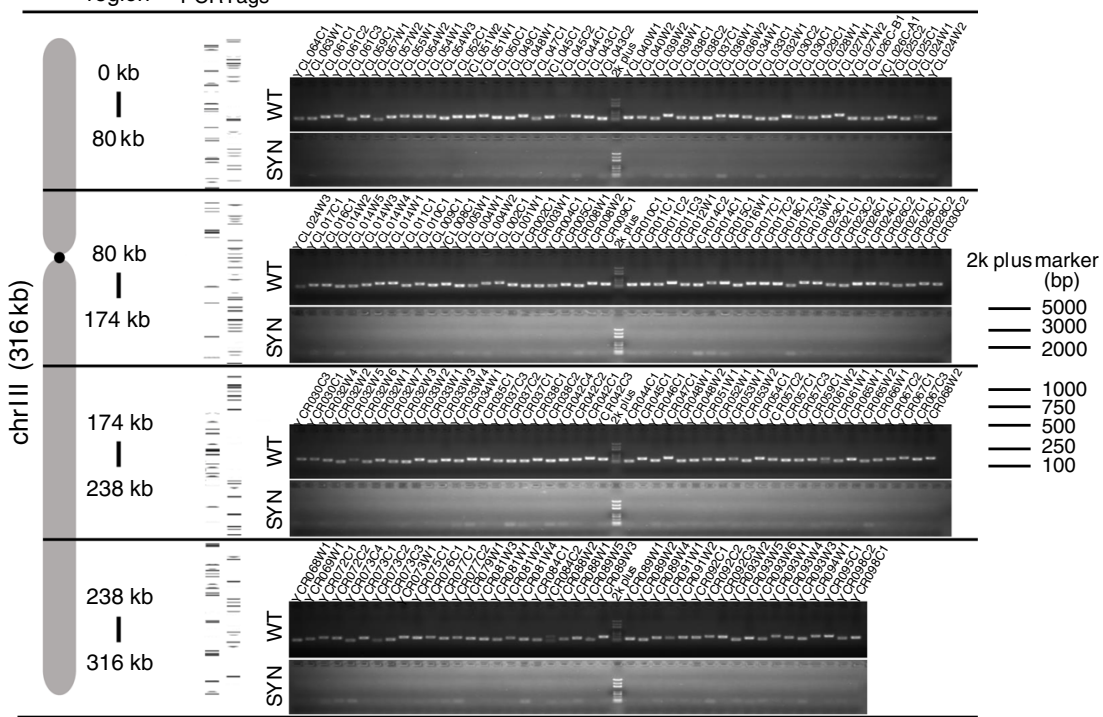

d
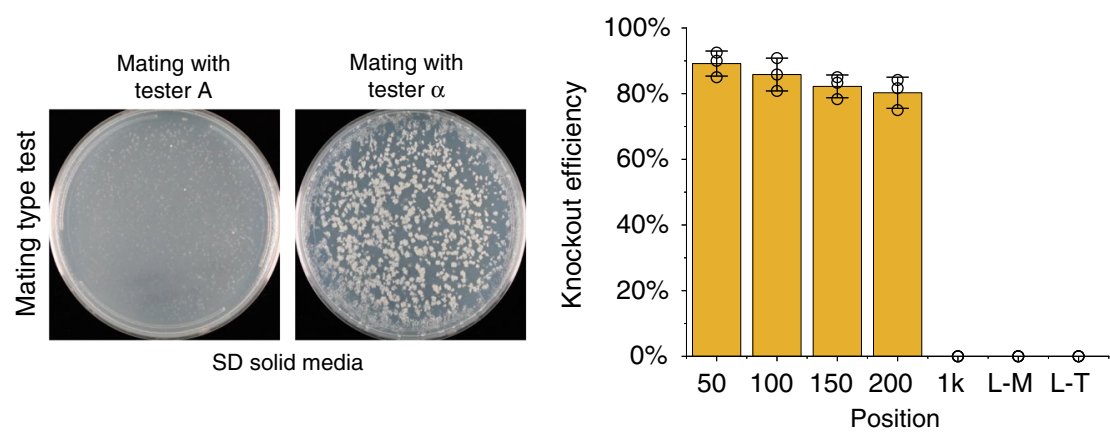

Fig. 1 Chromosome elimination by CRISPR-Cas9 induced one DSB near the centromere. a Mechanism for chromosome elimination via CRISPR-Cas9. The gray chromosome represents chrlll, and the red chromosome represents synlll. The CEN represents the centromere and the mosaic red chromosome represents the process of chromosome elimination in the diploid strain. $\mathbf{b}$ All synthetic and native PCRtags on synlll were tested. 372 synthetic and wildtype PCRtags were used to verify chromosome elimination of synIII in yHX0295. c Southern blot analysis of synlll elimination. The genomes of the BY4741, synIII, diploid (BY4741-synIII) were used as controls and four synlll elimination diploid strains (yHX0295, yHX0333, yHX0334, and yHX0334) were analyzed. All genomes were digested with HindIII. Chrlll yielded a 10,650 bp fragment, and synlll yielded a 4857 bp fragment after digestion and hybridization with the probe. The blue frame indicates fragments from chrlll, and the orange frame indicates fragments from synlll. d Mating type test of CRISPR-containing diploid strains by tester A and tester $\alpha$. SD solid media with tester A or tester $\alpha$ were mated to media with CRISPR-containing diploid strains. e Efficiency of chromosome elimination at different positions of synX. L-M represents the middle of the left arm, and L-T represents the telomere of the left arm. A total of 120 strains were tested for each replicate and the error bars indicate the standard deviations of all ( $n=3$ ) biological replicates. Source data are provided as a Source Data file.

confirmed the elimination of synX, which encodes RFP, and the endoreduplication of chrX, which encodes GFP (Fig. 2a).

Furthermore, the synthetic violacein pathway was used as a colorimetric phenotype to visualize the process of chromosome drive. With respect to the BY4742 strain, a six-gene-member violacein pathway (vio $\mathrm{A}, v i o \mathrm{~B}, v i o \mathrm{C}, v i o \mathrm{D}, v i o \mathrm{E}$, and $U R A 3$ ) spanning a region of $13717 \mathrm{bp}$ was inserted into the left arm of chrX, and the CRISPR-Cas9 expression cassette was inserted into the right arm of chrX (Supplementary Fig. 5). With respect to the synX strain, the synthetic CRISPR-targeted sequence (5'-GTTG CAAATGCTCCGTCGACGGG-3') was inserted $50 \mathrm{bp}$ from the centromere on the left $\overline{\text { arm }}$ of synX, preventing potential transmission in wild-type yeast. The BY4742 and synX strains described above were subsequently mated to induce the process of chromosome drive (Fig. 2b). To investigate the driven genotype of the progeny, the CRISPR-containing diploid strains were subjected to whole-genome sequencing. The results revealed that specific tag sequences of synX had been lost, and the sequencing depth of chrX was similar to that of the other chromosomes in the driven diploid strain yHX0296, verifying the elimination of the entire synX and the duplication of chrX (Fig. 2c, d).

To further investigate the driven phenotype of the progeny, CRISPR-containing diploid strains were randomly selected to undergo sporulation and tetrad dissection. Spores from the dissected tetrads were plated on synthetic complete (SC) media to visualize the color of colonies. A total of 70 individual diploid strains were randomly selected and tested. If the synX in the diploid strain was eliminated and the counterpart violacein-chrX was endoreduplicated, all four spores would display purple color, while the undriven diploids would undergo normal inheritance, producing two purple spores and two white spores per tetrad. The results showed that $87.14 \%(61 / 70)$ of the diploid strains inherited the violacein pathway, which was roughly equal to the efficiency of chromosome elimination for synX (Fig. 2e, Supplementary 
a
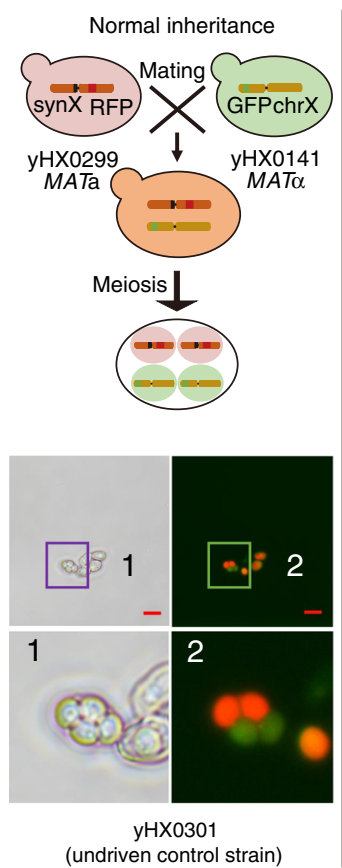

(undriven control strain)
Chromosome-driven inheritance
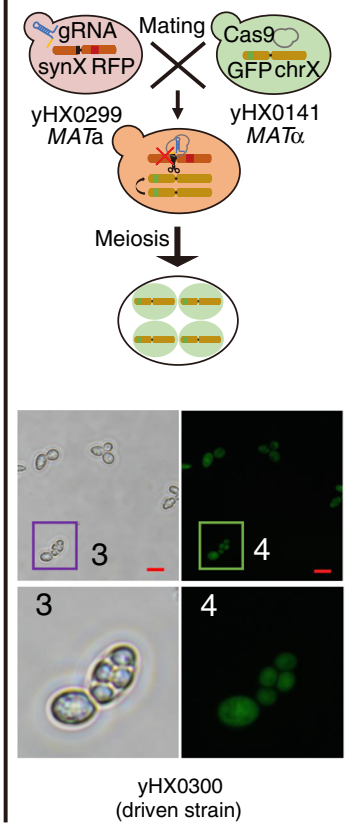

(driven strain)

b

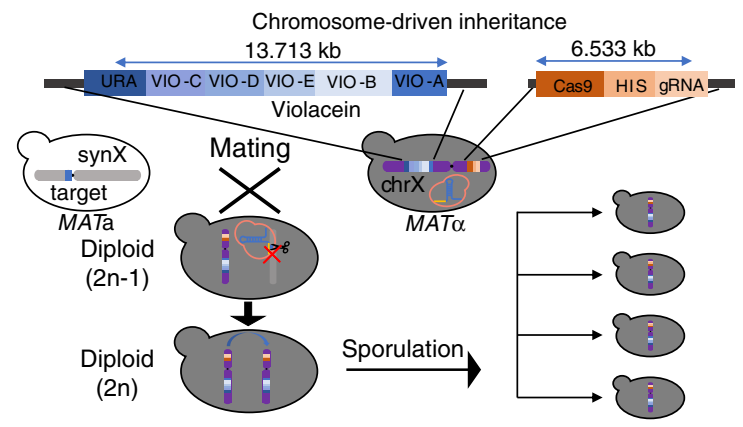

C

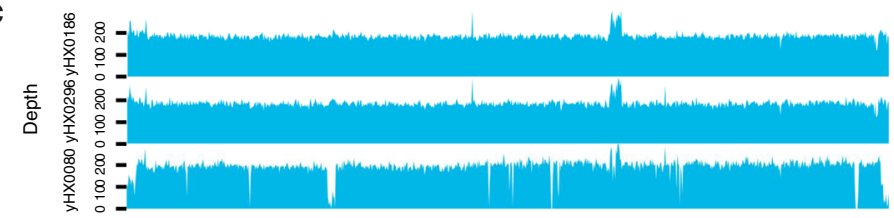

Position (bp) 22,077-22,104

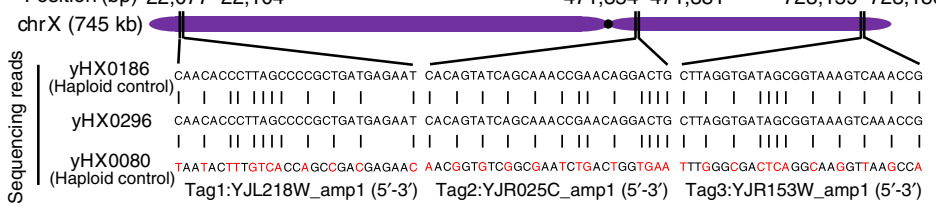

d

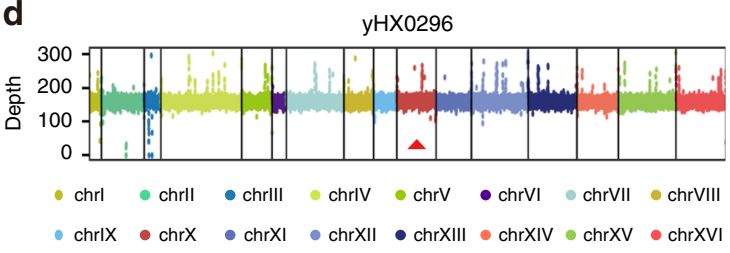

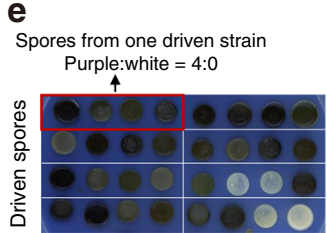

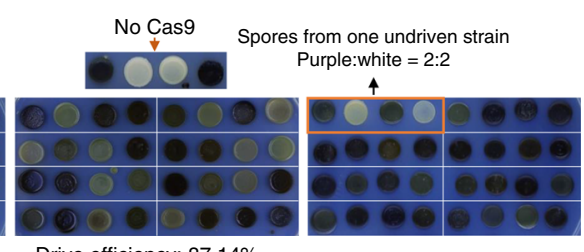

Drive efficiency: $87.14 \%$

Fig. 2 Chromosome drives with a synthetic violacein pathway. a Verification of chromosome endoreduplication via GFP and RFP in yeast. Strains were identified under visible light and observed for GFP and RFP. The haploid controls yHX0299 and yHX0141 displayed red and green color, respectively, under a fluorescence microscope. Three tetrads from the one driven strain, yHX0300, displayed a green color in all four spores. One tetrad was displayed at a higher resolution. One tetrad from the diploid control yHX0301 displayed two green spores and two red spores. Numbered squares indicate single tetrad shown at a higher resolution in the below panels. Scale bar, $10 \mu \mathrm{m}$. b Workflow of the chromosome-driven inheritance with components of the synthetic violacein pathway. c Whole-genome sequencing analysis of synX elimination in yHX0296. All the PCRTags in yHX0296 matched with the haploid control

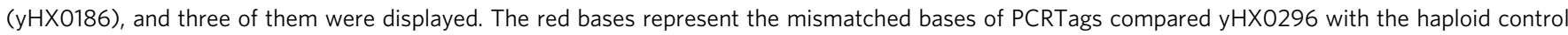
(yHX0080). d The coverage map of yHX0296. The driven diploid strain yHX0296 was used for the analysis of chromosome endoreduplication. The sequencing depths of all sixteen chromosomes were at the same scale. The sequencing depth of chrX was similar to that of the other chromosomes. The red triangle indicates the position of chrX in the coverage map. e Phenotypic test of spores from dissected tetrads. The spores with the synthetic violacein pathway produce purple colonies on SC media. In the absence of Cas9, normal 2:2 segregation was observed. A total of $87.14 \%$ of dissected tetrads were observed as having four purple spores. The spores of all 70 dissected tetrads are shown in Supplementary Fig. 6 . The red frame indicates four spores from one driven strain and the orange frame indicates four spores from one undriven strain. Source data are provided as a Source Data file.

Fig. 6 and Supplementary Table 3). To further verify chromosome elimination, spores from one driven diploid strain were tested for the elimination of synX. PCRTag analysis of four spores derived from one tetrad showed that 12 of the synthetic PCRTags across synX were lost in spores yHX0157, yHX0158, yHX0159, and yHX0160, indicating the loss of the entire synX (Supplementary Fig. 7). Pulsed-field gel electrophoresis (PFGE) analysis also revealed that synX was lost and that chrX (encoding all the components of the violacein pathway) were present in all four spores (Supplementary Fig. 8). In all 61 driven tetrads tested, four identical spores with violacein pathways were obtained from each tetrad, further verifying the duplication of violacein-chrX in the driven tetrads. We also tested the stability of the chromosome drive through multiple rounds of mating and sporulation. As shown in Supplementary Fig. 9, the purple-driven strain can transmit violacein-chrX to white yeast cells within three rounds of experiments.
Preferential transmission of complex genetic traits in yeast. Our results from the above experiment demonstrate that chromosome drive enables biased inheritance on a chromosomal scale in S. cerevisiae. How can we utilize chromosome drive compared with other self-propagating drive systems? Using quantitative trait locus (QTL) mapping and genome-wide association studies (GWASs), researchers have shown that many traits are polygenically influenced ${ }^{29}$. Additionally, recent developments in synthetic biology have enabled the redesign and synthesis of biosynthetic pathways and whole chromosomes $25,26,30,31$. Thus, we attempted to demonstrate the ability of chromosome drive to transmit complex genetic traits via megabase synthetic chromosome XII (synXII), heterogenous Y12 chromosome XIV (chrXIV) and Synthetic Chromosome Rearrangement and Modification by LoxP-mediated Evolution (SCRaMbLE)d synX in S. cerevisiae in subsequent experiments. 
With a highly repetitive ribosomal DNA (rDNA) gene cluster, chromosome XII (chrXII) is one of the largest chromosomes in the genome of $S$. cerevisiae. The internal transcribed spacer (ITS) is contained within the rDNA sequence and is considered the marker for species identification ${ }^{32}$. In a previous study, a megabase synXII of $S$. cerevisiae was chemically synthesized, and the rDNA was completely replaced by rDNA from Saccharomyces bayanus (S. bayanus) at the same position in JDY $476^{33}$. Here, we used chromosome drive to transmit the $S$. bayanus rDNA on synXII to S. cerevisiae (Fig. 3a). In our study, the CRISPR-Cas 9 expression cassette was inserted $242 \mathrm{~kb}$ from the centromere on the right arm of synXII in JDY476 cells (Supplementary Fig. 5). The synthetic CRISPR-targeted sequence (5'-GTTGCAAATGCTCCGTCGACGGG-3') was inserted $50 \mathrm{bp}$ from the centromere of chrXII in BY 4741 . The process of chromosome drive was initiated by mating the two strains described above. PCRTag analysis of randomly selected diploid strains revealed that chrXII was eliminated in 20.83\% (20/96) of the tested strains (Supplementary Table 3). Whole-genome sequencing analysis revealed that the wild-type PCRTags were lost and that the entire chrXII was eliminated in the diploid strains (yHX0297) (Supplementary Fig. 10). Owing to the difference in the ITS region between S. bayanus and S. cerevisiae, it can be used as a marker for synXII chromosome drive. Investigation of the driven spores via Sanger sequencing and restriction enzyme digestion of the PCR products of the ITS region indicated that the Apa I restriction site within the ITS region were no longer present in the tested driven strains (Fig. 3b, c). Thus, the rDNA in the driven strains had been swapped with the rDNA from $S$. bayanus by chromosome drive. These results indicated that chromosome drive can be used to transmit the specific chromosomal structures of repetitive rDNA gene cluster, resulting in changes in species identity.

Y12 is an industrial yeast strain used for fermentation owing to its thermotolerance ${ }^{34}$. However, it is difficult to dissect the explicit genetic mechanism underlying this thermotolerance since the strategy of adaptive evolution is commonly used to confer traits to industrial strains ${ }^{35,36}$. Here, using the strategy of chromosome elimination, we first attempted to map the thermotolerance trait at the chromosome scale in the heterozygous Y12-BY4741 diploid strain. Using a growth fitness test of haploid strains derived from the loss-of-heterozygosity (LOH) diploid strains at $40^{\circ} \mathrm{C}$, we found that chrXIV of Y12 contributes the most to thermotolerance (Supplementary Fig. 11). To further confirm whether the strain containing chrXIV of Y12 has a thermotolerance trait using chromosome transfer and elimination, we constructed a heterozygous haploid strain (yHX0266) in which chrXIV of BY4741 was replaced with chrXIV of Y12 (Supplementary Fig. 12). The results showed that $\mathrm{yHX} 0266$ was more thermotolerant than was BY4741, indicating that chrXIV of Y12 was the thermotolerance-related chromosome (Supplementary Fig. 13). To demonstrate the ability of chromosome drive to transmit the thermotolerance trait to other yeast strains, we attempted to transmit chrXIV of Y12 to laboratory yeast strain BY4742 via chromosome drive. As shown in Fig. 3d, yHX0266 was equipped with a driven CRISPR-Cas9 system, and the BY4742 strain was inserted with the synthetic CRISPRtargeted sequence (5'-GTTGCAAATGCTCCGTCGACGGG$\left.3^{\prime}\right) 50 \mathrm{bp}$ from the centromere of chrXIV (Supplementary Fig. 5). The process of chromosome drive was initiated by mating of the two strains as described above. Depending on the sequence differences between chrXIV of BY4742 and that of Y12, specific tags were designed to assess the elimination of the corresponding chromosome of BY4742 (Supplementary Fig. 14). PCR-based analysis revealed that of BY4742 chrXIV was eliminated in $40.63 \%(39 / 96)$ of the tested diploid strains (Supplementary Table 3). To verify the complete elimination of BY4742 chrXIV and the transmission of Y12 chrXIV into the driven spores, four driven spores (yHX0285, yHX0286, yHX0287, and yHX0288) from one diploid strain were tested by whole-genome sequencing. On the basis of the distribution of single-nucleotide polymorphisms (SNPs) throughout the entire chrXIV, the pattern of four driven spores was the same as that of Y12, indicating successful transmission of the entire Y12 chrXIV to the spores (Fig. 3e and Supplementary Table 4). Further, we tested the growth fitness of the four driven spores at 30,37 , and $40^{\circ} \mathrm{C}$ by serial dilution assays. The results showed that all four driven spores were obviously more thermotolerant than the BY4742 strain was, demonstrating the use of chromosome drive to transmit complex genetic traits in yeast (Fig. 3f).

As part of the Sc2.0 project, synthetic yeast chromosomes have been designed with hundreds of palindromic loxPsym sites located downstream of nonessential genes throughout the chromosomes ${ }^{25,26}$. Genomic segments between loxPsym sites can be rearranged with the expression of Cre recombinase, which leads to stochastic deletion, inversion, translocation, and duplication within and between synthetic chromosomes, which is referred to as the Synthetic Chromosome Rearrangement and Modification by LoxP-mediated Evolution (SCRaMbLE) system ${ }^{37-40}$. Under suitable screening conditions, SCRaMbLEd strains with improved traits can be generated 38,40 . However, owing to the process of totally random chromosomal rearrangement, it is still a challenge to dissect the structural variations that lead to specific phenotypic changes ${ }^{38-40}$. Here, we used chromosome drive to attempt to transmit the newly generated unresolved trait of chromium resistance. First, SCRaMbLEd synX strains were screened on SC media supplemented with $0.15 \mathrm{mM} \mathrm{Cd}$ $\left(\mathrm{NO}_{3}\right)_{2}$. Through a serial dilution assay, one SCRaMbLEd strain, yHX0065, was selected on the basis of its visible improvement in chromium resistance, and it was used as a drive carrier to transmit SCRaMbLEd synX to laboratory yeast BY4742 (Supplementary Fig. 15). The yHX0065 strain was equipped with the driven CRISPR-Cas9 system, and the BY4742 strain was inserted with the synthetic CRISPR-targeted sequence (5'-GTTGCAAAT GCTCCGTCGACGGG-3') 50 bp from the centromere of chrX (Supplementary Fig. 5). The process of chromosome drive was initiated by mating the two strains as described above (Fig. 3g). PCRTag analysis revealed that the target chromosome chrX was eliminated in $87.5 \%(84 / 96)$ of the tested diploid strains (Supplementary Table. 3). Whole-genome sequencing analysis revealed that the chromosome-elimination strain yHX0332 contained only synthetic PCRTags, verifying the elimination of chrX in yHX0332 (Supplementary Fig. 16). One driven diploid was selected and sporulated. Synthetic PCRTag analysis of the dissected spores showed that all four spores had been transmitted together with synX (Supplementary Fig. 17). Electrophoretic karyotype analysis of the dissected spores by PFGE revealed that all four spores were driven together with SCRaMbLEd synX, which was the same as that of strain yHX0065. It was also shown that SCRaMbLEd synX was longer than the original synX, indicating structural variation in synX induced by SCRaMbLE (Fig. 3h). To test the capability of chromium resistance of the driven spores, the four spores were subjected to a serial dilution assay on SC media supplemented with $0.15 \mathrm{mM} \mathrm{Cd}\left(\mathrm{NO}_{3}\right)_{2}$. The results showed that all driven spores grew better than the BY4742 strain did on the chromium media, indicating successful transmission of the unresolved phenotype of chromium resistance by chromosome drive (Fig. 3i). It was also indicated that the SCRaMbLEd synX of strain yHX0065 is directly related to chromium resistance. 


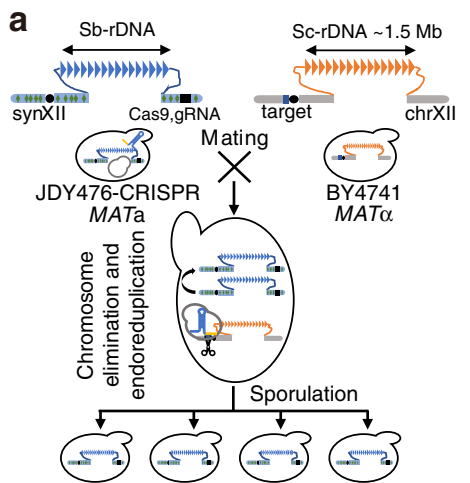

d

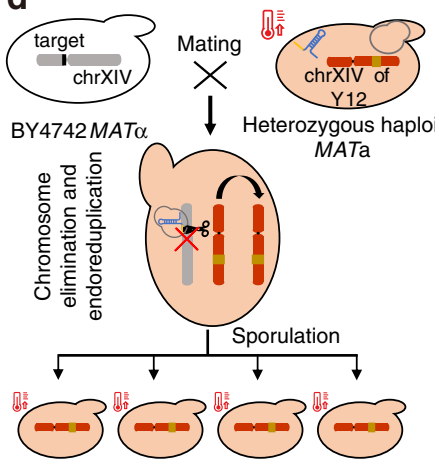

g

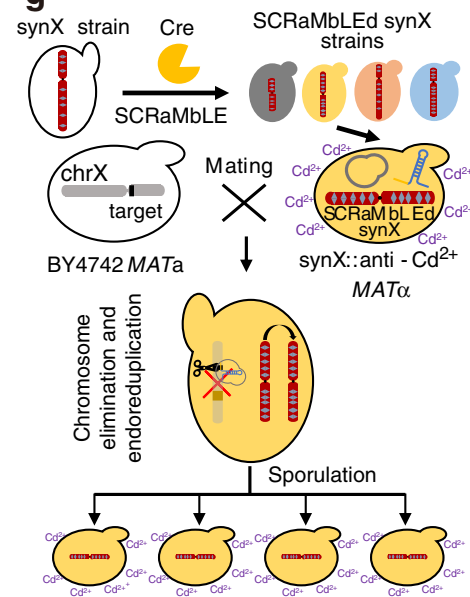

b

BY4741 $\operatorname{lmm}_{\text {AGCAAT GGGGGCOA GAGGTAA }}$

JDY476 SWWWhrwhm

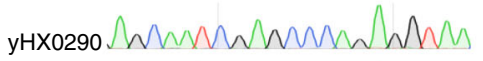

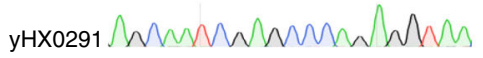

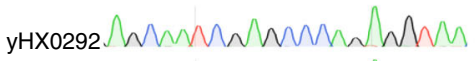

yHX0293 $\bigcap_{\text {AGCAATCGGGCCAA GAGGTAA }}$ c

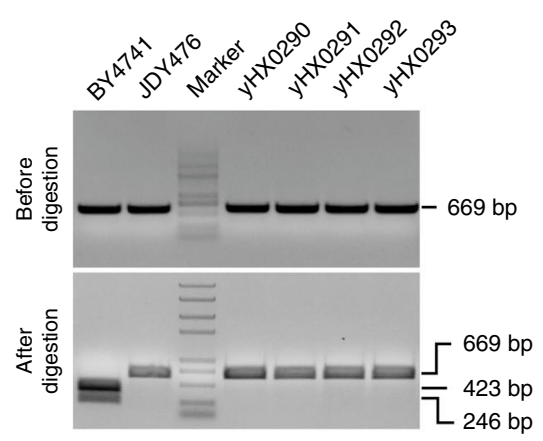

e

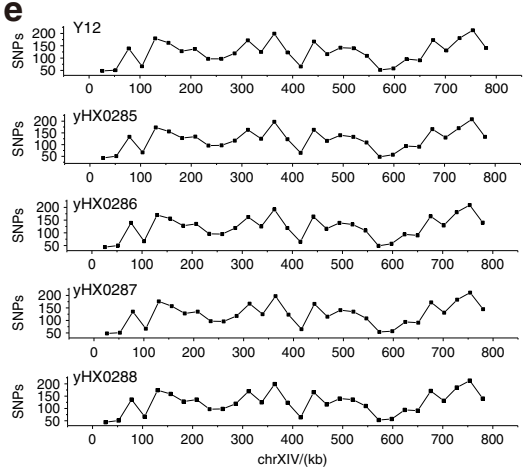

h

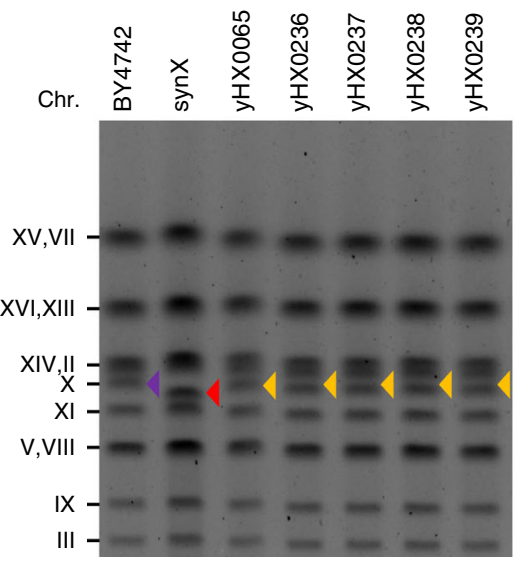

f

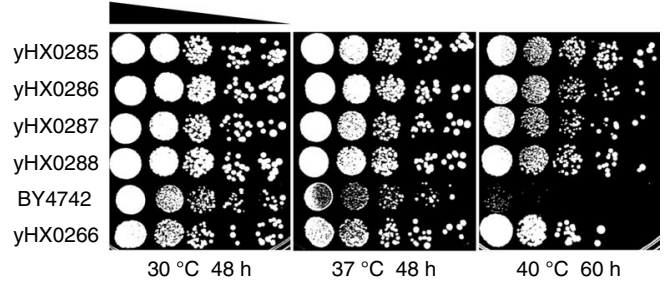

i

Fig. 3 Preferential transmission of complex genetic traits in yeast via chromosome drive. a Process by which chromosome drive transmits synXII by mating and sporulation. SynXII is encoded by a, highly repetitive rDNA sequence of S. bayanus. b Sequences of PCR products amplified from ITS regions for BY4741, JDY476 and four driven spores. The red frame indicates the sequence difference in rDNA between S. cerevisiae and S. bayanus. c Restriction enzyme digestion to verify the absence of the native ITS sequence in the driven spores. The PCR products were treated with (bottom) or without (top) Apal. Four driven spores (yHX0290, yHX0291, yHX0292, and yHX0293) was used for the test. BY4741 and JDY476 were used as negative and positive controls, respectively. $\mathbf{d}$ Process by which chromosome drive transmits thermotolerance-related chromosomes. The detailed process of the construction of the heterozygous haploid is shown in Supplementary Fig. 12. e Distribution of SNPs throughout the entire chrXIV for four driven spores (yHX0285,

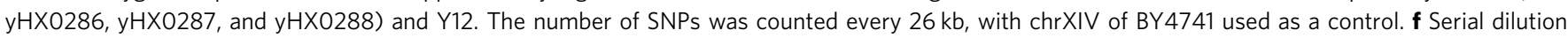

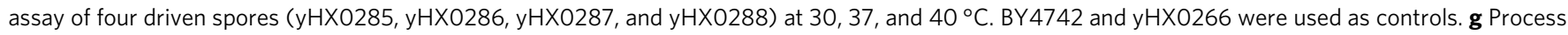
by which chromosome drive transmits SCRaMbLEd synX with an unresolved chromium resistance trait. $\mathbf{h}$ Electrophoretic karyotype analysis of four dissected spores (yHX0236, yHX0237, yHX238, and yHX0239) via PFGE. Strains BY4742, synX, and yHX0065 were used as controls for chrX, synX, and SCRaMbLEd synX, respectively. The purple triangle indicates the positions of chrX, the red triangle indicates the positions of synX and the yellow triangle

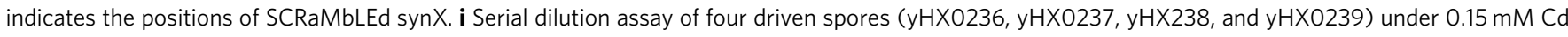
$\left(\mathrm{NO}_{3}\right)_{2}$ for $72 \mathrm{~h}$. Strains synX and BY4742 were used as controls. Source data are provided as a Source Data file.

\section{Discussion}

The chromosome drive system is designed to transmit a desired chromosome via sexual reproduction. Chromosome elimination is a vital step in the CRISPR-Cas9 chromosome drive system. In this study, we found that only one DSB near the centromere induced by CRISPR-Cas9 can effectively eliminate a chromosome in $S$. cerevisiae. In this species, DSB repair is based on mainly homology-directed repair (HDR) rather than nonhomologous 
end-joining (NHEJ) in the presence of a homologous repair template ${ }^{41}$. It was reported that the efficiency of HDR around centromeres is lower than that in other regions of chromosomes in S. cerevisiae ${ }^{42-45}$. Thus, we speculated that the CRISPR-Cas9induced DSB near the centromere may be hard to repair by HDR or NHEJ. The broken chromosome would be unstable and ultimately lost in the yeast cells. In other organisms, the contribution of HDR and NHEJ to DSB repair is quite different. In mammals, NHEJ is considered the major way to repair DSBs. For species with regional centromeres, previous studies have revealed that an entire chromosome can be eliminated by CRISPR-Cas9 targeting at multiple specific sites on the chromosome or in the centromeric region ${ }^{46-48}$. In addition, we also noticed that the elimination efficiency for different chromosomes varied in $S$. cerevisiae (Supplementary Table 3). The off-target mutations were then characterized via whole-genome sequencing. After analyzing ten individual off-target strains for each drive experiment of synX, chrXII, chrXIV, and chrX, we found that HDR happened in the CRISPR target region for the majority of the off-target strains in the experiments involving synX, chrXIV, and chrX. However, the CRISPR-targeted sequence remained intact for nine of the ten off-target strains in the drive experiment of chrXII (Supplementary Table 5). These results may be due to the low efficiency of CRISPR for chrXII, which contains a highly repetitive ribosomal gene cluster for the formation of a nucleolar structure, and there is also evidence that the efficiency of DSBs varies within different chromosomes ${ }^{45}$. Regarding of the existence off-target strains, it may be a problem for multiple generations of chromosome elimination. The chromosome drive system enables biased inheritance of an entire desired chromosome by elimination of the target chromosome and subsequent autonomous chromosome endoreduplication. In S. cerevisiae, chromosome endoreduplication is a recovery mechanism for the chromosome elimination ${ }^{28,49}$. Evidence has also shown that uniparental disomy (UPD), the phenomenon in which two copies of a chromosome from one parent are present and no copy from the other parent is present, occurs via monosomy rescue of chromosome $\mathrm{XIV}$ and chromosome $\mathrm{XV}^{50,51}$. However, in some cases, chromosome endoreduplication may not happen, and the mechanism underlying the chromosome endoreduplication essentially remains unknown ${ }^{28}$. To achieve the biased inheritance of desired chromosomes via the chromosome drive system, the process of chromosome endoreduplication may not be critical since elimination of the target chromosome in the zygotes results in inviable progeny without the desired chromosome. This process employs the same principle as that of CRISPR toxin-antidote drive, in which recessive lethal alleles are formed and half of offspring with recoded synthetic genes are rescued ${ }^{18,19,52}$.

Chromosome elimination via CRISPR-Cas9 is a powerful tool to induce whole-chromosomal $\mathrm{LOH}$ and can be developed as a method for mapping specific chromosome-related traits. In addition, chromosome elimination via CRISPR-Cas9 also provides a strategy for consolidating multiple synthetic yeast chromosomes into one cell by knocking out the counterpart wild-type chromosomes in synthetic-wild type heterozygous diploid strains, ultimately leading to the construction of a synthetic yeast genome $25,26,30,33,49$. With sexual reproduction and desired chromosome endoreduplication, the chromosome drive system can provide a method for the transmission of chromosome-related traits and various unresolved traits to facilitate genetic engineering of industrial strains. In this study, we demonstrate that the chromosome drive system enables biased inheritance of complex genetic traits or unresolved genetic traits on a chromosomal scale in yeast, extending possible applications of selfpropagating drive. With respect to the chromosome drive system in other given species, many studies on the efficiency of chromosome elimination, fertility and biosafety consequences should be performed.

\section{Methods}

Strains, plasmids, and primers. All the yeast strains used in this study are listed in Supplementary Data 1 the plasmids used are listed in Supplementary Data 2, and primers used are listed in Supplementary Data 3.

Yeast transformation. The yeast colonies were incubated in $5 \mathrm{~mL}$ of yeast peptone dextrose (YPD) overnight at $30^{\circ} \mathrm{C}$ with shaking at $220 \mathrm{rpm}$. Afterward, $200 \mu \mathrm{L}$ of the culture was transferred to $5 \mathrm{~mL}$ of YPD. When the $\mathrm{OD}_{600}$ reached $0.4-0.6,1 \mathrm{~mL}$ of the cells was harvested and washed twice with sterile $\mathrm{H}_{2} \mathrm{O}$. The pellets were subsequently treated with $1 \mathrm{~mL}$ of $0.1 \mathrm{M} \mathrm{LiOAc}$ solution and then gently placed on ice for 5-10 min. Afterward, $620 \mu \mathrm{L}$ of polyethylene glycol (PEG) 3350, $40 \mu \mathrm{L}$ of salmon DNA and $90 \mu \mathrm{L}$ of $1 \mathrm{M}$ LiOAc were mixed together, which served as transformation buffer. The salmon DNA was preheated at $100^{\circ} \mathrm{C}$ for $12 \mathrm{~min}$ and then placed on ice to cool. Pretreated cells $(150 \mu \mathrm{L})$ were immersed in transformation buffer and then mixed by being inverted up and down 7-10 times, after which they were cultured at $30^{\circ} \mathrm{C}$ for $30 \mathrm{~min}$. The culture was then mixed with dimethyl sulfoxide (DMSO) and heat-shocked at $42{ }^{\circ} \mathrm{C}$ for $18 \mathrm{~min}$. The solution was centrifuged at $1500 \times g$ for $2 \mathrm{~min}$, after which the supernatant was decanted. The cells were recovered and placed in $400 \mu \mathrm{L}$ of $5 \mathrm{mM} \mathrm{CaCl}_{2}$ for $7-10 \mathrm{~min}$ at room temperature, after which they were plated onto corresponding selective media.

Mating with tester A and tester $\boldsymbol{\alpha}$. Two different mating-type strains lacking HIS1, referred to as tester A and tester $\alpha$, were used. Both strains were selected and cultured overnight in $5 \mathrm{~mL}$ of YPD overnight at $30^{\circ} \mathrm{C}$ with shaking $220 \mathrm{rpm}$. Two hundred microliter of both culture solutions was plated onto SD solid media and recorded as test plates. Moreover, the diploid strains that remained to be tested for mating type were copied onto test plates, respectively. The SD solid media were cultured at $30^{\circ} \mathrm{C}$ for $24 \mathrm{~h}$ to observe the growth of the colonies. The mating type of Diploid strains that can grow on test plates was A, that are unable to grow on the test plates was $\mathrm{A} / \boldsymbol{\alpha}$.

Yeast mating process. Yeasts with different mating types were incubated in corresponding selective media overnight. $1 \mathrm{~mL}$ of yeasts with different mating types was harvested and washed twice with sterile $\mathrm{ddH}_{2} \mathrm{O}$ twice. Two hundred microliter of each culture was then added to $5 \mathrm{~mL}$ of YPD (in the YPD, each strain grew well) and incubated at $30^{\circ} \mathrm{C}$ with shaking at $220 \mathrm{rpm}$ for $8 \mathrm{~h}$. One milliliter of the mating solution was subsequently harvested and washed with sterile $\mathrm{ddH}_{2} \mathrm{O}$ twice. Ten microliters of solution and $200 \mu \mathrm{L}$ of sterile $\mathrm{ddH}_{2} \mathrm{O}$ were mixed together, plated onto selective media, and then incubated at $30^{\circ} \mathrm{C}$ for $2-3$ days.

PCRTag analysis of chromosome elimination. PCRTags located at both ends of the chromosome and at both sides of the centromere on each chromosome were selected as markers to assess the efficiency of syn X, synIII, chrX, synXII, and chrXIV elimination. The loss of PCRTags of synX, chrX (YJL219W_amp1, YJL002C_amp1, YJR001W_amp2, YJR155W_amp1), synIII (YCL064C_amp1, YCL001W_amp1, YCR002C_amp1, YCR098C_amp1), and synXII (YLL048C_amp1, YLL001W_amp1, YLR001C_amp1, YLR455W_amp1) can be used to assess the elimination of chromosomes. With respect to the efficiency of chrXIV elimination, PCRTags (Y12-left, Y12-right) were designed to test chromosome elimination.

Meiosis and sporulation. Yeasts were incubated in $5 \mathrm{~mL}$ of YPD overnight at $30^{\circ} \mathrm{C}$ with shaking at $220 \mathrm{rpm}$, after which $200 \mu \mathrm{L}$ of the culture was transferred to $5 \mathrm{~mL}$ of $2 \times$ YPD and then incubated at $30^{\circ} \mathrm{C}$ with shaking at $220 \mathrm{rpm}$ for $10 \mathrm{~h}$. All the cells were harvested and washed with sterile $\mathrm{ddH}_{2} \mathrm{O}$ three times (the YPD was washed away totally to ensure the efficiency of sporulation). One milliliter of 50× sporulation media, $500 \mu \mathrm{L}$ of the required amino acids (100×; uracil, histidine, leucine, or tryptophan), and $150 \mu \mathrm{L}$ of $10 \%$ yeast extract were mixed together and then diluted with sterile $\mathrm{ddH}_{2} \mathrm{O}$ to a volume of $50 \mathrm{~mL}$. All washed cells were transferred into sporulation solution and mixed well. The culture was incubated at $25^{\circ} \mathrm{C}$ with shaking at $200 \mathrm{rpm}$ for 3-6 days. The presence of tetrads was subsequently observed with a microscope.

Serial dilution assays for selections. Yeasts were incubated in $5 \mathrm{~mL}$ of YPD overnight at $30^{\circ} \mathrm{C}$ with shaking at $220 \mathrm{rpm}$, after which $200 \mu \mathrm{L}$ of the culture was transferred to $5 \mathrm{~mL}$ of YPD at $30^{\circ} \mathrm{C}$ with shaking at $220 \mathrm{rpm}$ and then incubated to an $\mathrm{OD}_{600}$ of 1 . The solution was serially diluted in 10 -fold increments in $\mathrm{ddH}_{2} \mathrm{O}$ five times, and $4 \mu \mathrm{L}$ of the diluted solution was plated from lowest to highest concentration on the corresponding media. When the solution was dry, the media were maintained at a suitable temperature for 2-3 days.

Pulsed-field gel electrophoresis (PFGE). The PFGE protocol was modified ${ }^{53}$, and a single colony was inoculated into $5 \mathrm{~mL}$ of YPD overnight, with shaking at 
$30{ }^{\circ} \mathrm{C}$. One milliliter of the overnight culture was transferred to a tube and was centrifuged at $1200 \times g$ for $2 \mathrm{~min}$ at room temperature. Cells were washed twice in solution I ( $0.05 \mathrm{M}$ EDTA, $0.01 \mathrm{M}$ Tris, $\mathrm{pH} 7.5)$ and resuspend in $150 \mu \mathrm{L}$ solution I with $10 \mu \mathrm{L}$ zymolyase $\left(2 \mathrm{mg} \mathrm{mL}^{-1}\right.$ zymolyase $20 \mathrm{~T}, 10 \mathrm{mM}$ Sodium Phosphate, $\mathrm{pH}$ 7.5). Cells were placed in the $42^{\circ} \mathrm{C}$ heat block. Two hundred and fifty microliter of agarose solution $\left(1 \%\left(\mathrm{w} \mathrm{v}^{-1}\right)\right.$ low melting temp agarose, $\left.0.125 \mathrm{M} \mathrm{EDTA}, \mathrm{pH} 7.5\right)$ was pre-incubated at $42{ }^{\circ} \mathrm{C}$ and mixed with cells by pipetting with a wide bore pipette tip in the tube. The tube was placed on ice immediately and added $400 \mu \mathrm{L}$ of LET (0.5 M EDTA, $0.01 \mathrm{M}$ Tris, pH 7.5). The tube was incubated for 8-10 h overnight at $37^{\circ} \mathrm{C}$, and placed on ice for $10-20 \mathrm{~min}$, then transferred the agarose plug in the tube to a $15 \mathrm{ml}$ falcon tube. Four hundred microliter of NDS $(0.5 \mathrm{M}$ EDTA, $0.01 \mathrm{M}$ Tris, $1 \%\left(\mathrm{w} \mathrm{v}^{-1}\right)$ Sodium Lauryl Sarcosine, $2 \mathrm{mg} \mathrm{mL}^{-1}$ proteinase $\mathrm{K}$, $\mathrm{pH}$ 7.5) was added and incubated overnight at $50^{\circ} \mathrm{C}$. The tubes was placed on ice for $10 \mathrm{~min}$. The NDS was exchanged for solution I and rock/swirl gently at room temperature for $1 \mathrm{~h}$ and repeated the wash three more times. Plugs were stored in fresh solution I at $4^{\circ} \mathrm{C}$. The electrophoresis process was performed in one stage, and the gel was made with $1 \%$ low-melting agarose and $1 \times$ Tris/borate/EDTA (TBE) buffer. The electrophoresis conditions were as follows: switch time of $60-120 \mathrm{~s}$, run time of $20 \mathrm{~h}$, angle of $120^{\circ}$ and voltage of $6 \mathrm{~V} \mathrm{~cm}^{-1}$.

Fluorescence detection of tetrads. The strains were sporulated prior to observations. Ten microliters of sporulated culture was mounted onto a slide, which was then sealed with a coverslip. The fluorescence of the tetrads was scored via a Nikon Eclipse Ci-L Scope equipped with Nikon Plan Fluor $40 \times / 0.75$ objective with Nikon DAPI-FITC filter and Nikon TRITC filters. The tetrads were observed under visible light and then scored for RFP and GFP. The images were taken under suitable light conditions.

\section{Thermotolerance-related chromosomes mapping. The synthetic CRISPR-} targeted sequence (5'-GTTGCAAATGCTCCGTCGACGGG-3') was inserted 50 bp from the centromere of each chromosome of BY4741. In addition, URA3 was integrated into chrIII in each strain. In the Y12 strain $(M A T \alpha)$, components of the CRISPR-Cas9 system were inserted into chrXIV. The two strains described above were mated and plated on solid SC-URA-LEU media, after which they were cultured at $30^{\circ} \mathrm{C}$ for 3-5 days. Single colonies were selected to test chromosome elimination in the heterozygous Y12-BY4741 diploid strain via PCR-based analysis. The PCRTags used are described in Supplementary Table. 8. The chromosomeeliminated diploid strains were selected for sporulation and dissection into spores. The spores were tested for their thermotolerance with Y12 and BY4741 via a serial dilution assay at 30 and $40{ }^{\circ} \mathrm{C}$. The spores that contained thermotolerance-related chromosomes of Y12 grew better than the controls did at $40^{\circ} \mathrm{C}$.

Construction of a thermotolerant heterozygous haploid. The karI gene is an essential gene in S. cerevisiae. In yeast, the karI mutation causes a lack of nuclear fusion. When one of the mating partners has a karI mutation, nuclear fusion is unable to occur ${ }^{54}$. First, we constructed a karI mutation strain to transfer chromosomes. We deleted karI followed by the complement of the plasmid containing karI in BY4741. For the plasmid containing karI, prs415 was extracted from Escherichia coli, and the karI DNA segment was amplified from the yeast genome. Both of them had NotI and BamHI restriction sites and were digested. The karI DNA segment was fused to the prs415 carrier using T4 DNA ligase to construct the plasmid. The karI-deleted segment was constructed together with upstream and downstream homologous arms and the URA3 marker. The deleted segment and plasmid were cotransformed into BY4741, which was then plated onto solid SCURA-LEU media. The media were subsequently incubated at $30^{\circ} \mathrm{C}$ for $2-3$ days. The transformed strains were verified by PCR, and the correct strain was selected for subsequent construction. The karI 15 segment was transformed into the positive strain, plated on solid SC +5 -FOA media where strains lacking the URA3 marker can grow, and then incubated at $30^{\circ} \mathrm{C}$ for $2-4$ days. Colonies that grew on solid SC + 5-FOA were analyzed via PCR, and the PCR products of the colonies were aligned with the sequence of karI 15 to obtain the karI $\Delta 15$ mutation strain (yHX0230).

In yHX0230, the CRISPR-targeted sequence (5'-GTTGCAAATGCTCCGT CGACGGG-3') was inserted $50 \mathrm{bp}$ from the centromere on the left arm of chrXIV by the use of the URA3 marker, and the LEU2 marker was integrated into chromosome XI. In addition, can-100 was deleted in yHX0230. In the Y12 strain yHX0192 (MAT $\alpha$ ), the CRISPR-Cas9 system was inserted into chrXIV. Afterward, yHX0230 and yHX0192 were mated, and all the mated cells were plated onto solid SC-URA-LEU+ canavanine media. The media were then cultured at $30^{\circ} \mathrm{C}$ for 3-5 days. Single colonies were selected to test the mating type and the loss of chrXIV in BY4741 via PCR-based analysis. In heterozygous haploids, the mating type was MATa, and chrXIV of BY4741 was eliminated.

Whole-genome sequencing. The strain samples were prepared for whole-genome sequencing according to GENEWIZ's standard preparation protocol. The samples were sent on dry ice to GENEWIZ in Suzhou. The sequencing data were analyzed and inductively charted for easy analysis.
SCRaMbLE of synX strain to generate chromium-resistance. The Cre-EBD plasmid, in which an engineered Cre recombinase was fused to the murine estrogen-binding domain, was used to prevent Cre expressed in the absence of $\beta$ estradiol and the activity of SCRaMbLE. The synX strains carrying the Cre-EBD plasmid were incubated in $5 \mathrm{~mL}$ of SC-HIS media overnight at $30^{\circ} \mathrm{C}$ and $220 \mathrm{rpm}$ Five microliter of $\beta$-estradiol $(1 \mathrm{mM})$ and the culture was were then mixed together and cultured at $30^{\circ} \mathrm{C}$ with shaking at $220 \mathrm{rpm}$ for $8 \mathrm{~h}$. One microliter of the cells was harvested and washed with $\mathrm{ddH}_{2} \mathrm{O}$ twice to remove all the estradiol. The solution was subsequently diluted 1000 -fold, and $100 \mu \mathrm{L}$ of the diluted solution was plated onto solid SC media supplemented with $0.15 \mathrm{mM} \mathrm{Cd}\left(\mathrm{NO}_{3}\right)_{2}$. The media were then cultured at $30^{\circ} \mathrm{C}$ for $3-5$ days, after which the colony sizes were measured.

Reporting summary. Further information on research design is available in the Nature Research Reporting Summary linked to this article.

\section{Data availability}

All sequencing data that support the findings of this study have been deposited into GeneBank that are available from the Sequence Read Archive under Accession Code SRP269526. Source data are provided with this paper. Any other relevant data are available from the authors upon reasonable request.

Received: 9 March 2020; Accepted: 6 August 2020; Published online: 28 August 2020

\section{References}

1. Werren, J. H. Selfish genetic elements, genetic conflict, and evolutionary innovation. Proc. Natl Acad. Sci. 108, 10863-10870 (2011).

2. Doolittle, W. F. \& Sapienza, C. Selfish genes, the phenotype paradigm and genome evolution. Nature 284, 601-603 (1980).

3. Thorner, J. \& Gimble, F. S. Homing of a DNA endonuclease gene by meiotic gene conversion in Saccharomyces cerevisiae. Nature 357, 301-306 (1992).

4. Hurst, G. D. D. \& Werren, J. H. The role of selfish genetic elements in eukaryotic evolution. Nat. Rev. Genet. 2, 597-606 (2001).

5. Austin, B. U. R. T., Trivers, R. \& Burt, A. Genes in Conflict: The Biology of Selfish Genetic Elements (Harvard University Press, Harvard, 2009).

6. Werren, J. H., Nur, U. \& Wu, C. I. Selfish genetic elements. Trends Ecol. Evol. 3, 297-302 (1988).

7. Meiklejohn, C. D. \& Tao, Y. Genetic conflict and sex chromosome evolution. Trends Ecol. Evol. 25, 215-223 (2010).

8. Champer, J., Buchman, A. \& Akbari, O. S. Cheating evolution: engineering gene drives to manipulate the fate of wild populations. Nat. Rev. Genet. 17, 146-159 (2016).

9. Champer, J. et al. Molecular safeguarding of CRISPR gene drive experiments. eLife 8, e41439 (2019).

10. DiCarlo, J. E., Chavez, A., Dietz, S. L., Esvelt, K. M. \& Church, G. M. Safeguarding CRISPR-Cas9 gene drives in yeast. Nat. Biotechnol. 33, 1250-1255 (2015)

11. Shapiro, R. S. et al. A CRISPR-Cas9-based gene drive platform for genetic interaction analysis in Candida albicans. Nat. Microbiol. 3, 73-82 (2018).

12. Kyrou, K. et al. A CRISPR-Cas9 gene drive targeting doublesex causes complete population suppression in caged Anopheles gambiae mosquitoes. Nat. Biotechnol. 36, 1062-1066 (2018).

13. Champer, J. et al. Novel CRISPR/Cas9 gene drive constructs reveal insights into mechanisms of resistance allele formation and drive efficiency in genetically diverse populations. PLoS Genet. 13, e1006796 (2017).

14. Chan, Y., Naujoks, D. A., Huen, D. S. \& Russell, S. Insect population control by homing endonuclease-based gene drive: an evaluation in Drosophila melanogaster. Genetics 188, 33-44 (2011).

15. Champer, J. et al. CRISPR gene drive efficiency and resistance rate is highly heritable with no common genetic loci of large effect. Genetics 212, 333-341 (2019).

16. Grunwald, H. A. et al. Super-Mendelian inheritance mediated by CRISPRCas9 in the female mouse germline. Nature 566, 105-109 (2019).

17. Lindholm, A. K. et al. The ecology and evolutionary dynamics of meiotic drive. Trends Ecol. Evol. 31, 315-326 (2016).

18. Champer, J., Kim, I. K., Champer, S. E., Clark, A. G. \& Messer, P. W. Performance analysis of novel toxin-antidote CRISPR gene drive systems. BMC Biol. 18, 1-17 (2020).

19. Champer, J. et al. A toxin-antidote CRISPR gene drive system for regional population modification. Nat. Commun. 11, 1-10 (2020).

20. Shao, Y. et al. Creating a functional single-chromosome yeast. Nature $\mathbf{5 6 0}$ 331-335 (2018).

21. Carbon, J. Yeast centromeres: structure and function. Cell 37, 351-353 (1984) 
22. Lefrancois, P., Auerbach, R. K., Yellman, C. M., Roeder, G. S. \& Snyder, M Centromere-like regions in the budding yeast genome. PLoS Genet. 9, e1003209 (2013).

23. Clarke, L. \& Carbon, J. Isolation of a yeast centromere and construction of functional small circular chromosomes. Nature 287, 504-509 (1980).

24. Costanzo, M. et al. A global genetic interaction network maps a wiring diagram of cellular function. Science 353 (2016).

25. Wu, Y. et al. Bug mapping and fitness testing of chemically synthesized chromosome X. Science 355 (2017).

26. Xie, Z. et al. "Perfect" designer chromosome V and behavior of a ring derivative. Science 355, f4704 (2017).

27. Annaluru, N. et al. Total synthesis of a functional designer eukaryotic chromosome. Science 344, 55-58 (2014).

28. Reid, R. J. D. et al. Chromosome-scale genetic mapping using a set of 16 conditionally stable Saccharomyces cerevisiae chromosomes. Genetics 180, 1799-1808 (2008).

29. Frazer, K. A., Murray, S. S., Schork, N. J. \& Topol, E. J. Human genetic variation and its contribution to complex traits. Nat. Rev. Genet. 10, 241-251 (2009).

30. Mercy, G. et al. 3D organization of synthetic and scrambled chromosomes. Science 355, f4597 (2017).

31. Galanie, S., Thodey, K., Trenchard, I. J., Interrante, M. F. \& Smolke, C. D. Complete biosynthesis of opioids in yeast. Science 349, 1095-1100 (2015).

32. Wayne Litaker, R. et al. Recognizing dinoflagellate species using ITS rDNA sequences. J. Phycol. 43, 344-355 (2007).

33. Zhang, W. et al. Engineering the ribosomal DNA in a megabase synthetic chromosome. Science 355, f3981 (2017).

34. Dandi, N. D., Dandi, B. N. \& Chaudhari, A. B. Bioprospecting of thermo- and osmo-tolerant fungi from mango pulp-peel compost for bioethanol production. Antonie van. Leeuwenhoek 103, 723-736 (2013).

35. Satomura, A., Miura, N., Kuroda, K. \& Ueda, M. Reconstruction of thermotolerant yeast by one-point mutation identified through whole-genome analyses of adaptively-evolved strains. Sci. Rep. 6, 1-9 (2016)

36. Caspeta, L. \& Nielsen, J. Thermotolerant yeast strains adapted by laboratory evolution show trade-off at ancestral temperatures and preadaptation to other stresses. Mbio 6, e431 (2015).

37. Jia, B. et al. Precise control of SCRaMbLE in synthetic haploid and diploid yeast. Nat. Commun. 9, 1-13 (2018)

38. Shen, M. J. et al. Heterozygous diploid and interspecies SCRaMbLEing. Nat. Commun. 9, 1-8 (2018).

39. $\mathrm{Wu}, \mathrm{Y}$. et al. In vitro DNA SCRaMbLE. Nat. Commun. 9, 1-9 (2018).

40. Wang, J. et al. Ring synthetic chromosome V SCRaMbLE. Nat. Commun. 9, 3783 (2018).

41. Clikeman, J. A., Khalsa, G. J., Barton, S. L. \& Nickoloff, J. A. Homologous recombinational repair of double-strand breaks in yeast is enhanced by MAT heterozygosity through yKU-dependent and -independent mechanisms. Genetics 157, 579-589 (2001).

42. Gerton, J. L. et al. Global mapping of meiotic recombination hotspots and coldspots in the yeast Saccharomyces cerevisiae. Proc. Natl Acad. Sci. 97, 11383-11390 (2000)

43. Petes, T. D. Meiotic recombination hot spots and cold spots. Nat. Rev. Genet. 2, 360-369 (2001).

44. Pan, J. et al. A hierarchical combination of factors shapes the genome-wide topography of yeast meiotic recombination initiation. Cell 144, 719-731 (2011).

45. Blitzblau, H. G., Bell, G. W., Rodriguez, J., Bell, S. P. \& Hochwagen, A. Mapping of meiotic single-stranded DNA reveals double-strand-break hotspots near centromeres and telomeres. Curr. Biol. 17, 2003-2012 (2007).

46. Zuo, E. et al. CRISPR/Cas9-mediated targeted chromosome elimination. Genome Biol. 18, 224 (2017).

47. Galizi, R. et al. A synthetic sex ratio distortion system for the control of the human malaria mosquito. Nat. Commun. 5, 3977 (2014).

48. Adikusuma, F., Williams, N., Grutzner, F., Hughes, J. \& Thomas, P. Targeted deletion of an entire chromosome using CRISPR/Cas9. Mol. Ther. 25, 1736-1738 (2017).
49. Mitchell, L. A. et al. Synthesis, debugging, and effects of synthetic chromosome consolidation: synVI and beyond. Science 355, f4831 (2017)

50. Conlin, L. K. et al. Mechanisms of mosaicism, chimerism and uniparental disomy identified by single nucleotide polymorphism array analysis. Hum. Mol. Genet. 19, 1263-1275 (2010).

51. de Nooij-van Dalen, A. G., van Buuren-van Seggelen, V. H., Lohman, P. H. \& Giphart-Gassler, M. Chromosome loss with concomitant duplication and recombination both contribute most to loss of heterozygosity in vitro. Genes Chromosomes Cancer 21, 30-38 (1998).

52. Champer, J., Champer, S. E., Kim, I., Clark, A. G. \& Messer, P. W. Design and analysis of CRISPR-based underdominance toxin-antidote gene drives. Preprint at https://www.biorxiv.org/content/10.1101/861435v2 (2019).

53. Herschleb, J., Ananiev, G. \& Schwartz, D. C. Pulsed-field gel electrophoresis. Nat. Protoc. 2, 677-684 (2007).

54. Torres, E. M. et al. Effects of aneuploidy on cellular physiology and cell division in haploid yeast. Science 317, 916-924 (2007).

\section{Acknowledgements}

We are grateful to Junbiao Dai for providing synXII strain, Jef D. Boeke and Srinivasan Chandrasegaran for providing synIII strain. This work was supported by the National Key Research and Development Program of China (2018YFA0900100), the National Natural Science Foundation of China (31971351 and 21750001) and Young Elite Scientist Sponsorship Program by CAST (YESS) (2018QNRC001).

\section{Author contributions}

Y.W. and Y.J.Y. conceived the overall project; H.X. and Y.W. designed the experiments; H.X., M.Z.H., and S.Y.Z. performed the experiments; H.X. and M.Z.H. performed bioinformatic analysis; H.X., B.Z.L., Y.J.Y., and Y.W. wrote the paper, with input from all authors.

\section{Competing interests}

The authors declare no competing interests.

\section{Additional information}

Supplementary information is available for this paper at https://doi.org/10.1038/s41467 020-18222-0.

Correspondence and requests for materials should be addressed to Y.W.

Peer review information Nature Communications thanks the anonymous reviewer(s) for their contribution to the peer review of this work. Peer reviewer reports are available.

Reprints and permission information is available at http://www.nature.com/reprints

Publisher's note Springer Nature remains neutral with regard to jurisdictional claims in published maps and institutional affiliations.

Open Access This article is licensed under a Creative Commons Attribution 4.0 International License, which permits use, sharing, adaptation, distribution and reproduction in any medium or format, as long as you give appropriate credit to the original author(s) and the source, provide a link to the Creative Commons license, and indicate if changes were made. The images or other third party material in this article are included in the article's Creative Commons license, unless indicated otherwise in a credit line to the material. If material is not included in the article's Creative Commons license and your intended use is not permitted by statutory regulation or exceeds the permitted use, you will need to obtain permission directly from the copyright holder. To view a copy of this license, visit http://creativecommons.org/ licenses/by/4.0/.

(c) The Author(s) 2020 